\title{
Identification of MicroRNAs as Potential Blood-Based Biomarkers for Diagnosis and Therapeutic Monitoring of Active Tuberculosis
}

\author{
Junseong Kim ${ }^{1,2, \dagger}$, Heechul Park ${ }^{1,2,+} \mathbb{D}$, Sung-Bae Park ${ }^{1,2}$, Eun Ju Lee ${ }^{1,2}$, Min-A Je ${ }^{1,2}$, Eunsol Ahn ${ }^{3}$, Bora Sim ${ }^{4}$, \\ Jiyoung Lee ${ }^{1}$, Hyunwoo Jin ${ }^{1,2} \mathbb{D}$, Kyung Eun Lee ${ }^{1,2} \mathbb{D}$, Sang-Nae Cho ${ }^{3,4}$, Young Ae Kang ${ }^{5}$, Hyejon Lee ${ }^{3, *}$, \\ Sunghyun $\operatorname{Kim}^{1,2, * \mathbb{D}}$ and Jungho Kim ${ }^{1, *}$
}

check for updates

Citation: Kim, J.; Park, H.; Park, S.-B.; Lee, E.J.; Je, M.-A.; Ahn, E.; Sim, B.; Lee, J.; Jin, H.; Lee, K.E.; et al. Identification of MicroRNAs as Potential Blood-Based Biomarkers for Diagnosis and Therapeutic Monitoring of Active Tuberculosis. Diagnostics 2022, 12, 369. https://doi.org/10.3390/ diagnostics12020369

Academic Editor: Xavier Bosch

Received: 28 December 2021

Accepted: 29 January 2022

Published: 1 February 2022

Publisher's Note: MDPI stays neutral with regard to jurisdictional claims in published maps and institutional affiliations.

Copyright: (C) 2022 by the authors. Licensee MDPI, Basel, Switzerland. This article is an open access article distributed under the terms and conditions of the Creative Commons Attribution (CC BY) license (https:// creativecommons.org/licenses/by/ $4.0 /)$.
1 Department of Clinical Laboratory Science, College of Health Sciences, Catholic University of Pusan, Busan 46252, Korea; wnstjd028@naver.com (J.K.); wt2626@naver.com (H.P.); tjdqo2013@naver.com (S.-B.P.); leeeunju41@naver.com (E.J.L.); je.mina0504@gmail.com (M.-A.J.); lab84@hanmail.net (J.L.); jjinhw@cup.ac.kr (H.J.); kelee@cup.ac.kr (K.E.L.)

2 Clinical Trial Specialist Program for In Vitro Diagnostics, Brain Busan 21 Plus Program, Graduate School, Catholic University of Pusan, Busan 46252, Korea

3 Clinical Vaccine Research Section, International Tuberculosis Research Center, Seoul 03772, Korea; eunsol486@naver.com (E.A.); RAYCHO@yuhs.ac (S.-N.C.)

4 Department of Microbiology, Institute of Immunology and Immunological Disease, Yonsei University College of Medicine, Seoul 03772, Korea; SIMBR0129@yuhs.ac

5 Division of Pulmonary and Critical Care Medicine, Department of Internal Medicine, Severance Hospital, Institute of Immunology and Immunological Disease, Yonsei University College of Medicine, Seoul 03772, Korea; MDKANG@yuhs.ac

* Correspondence: hyejonlee@gmail.com (H.L.); shkim0423@cup.ac.kr (S.K.); jutosa70@cup.ac.kr (J.K.); Tel.: +82-2-2228-0772 (H.L.); +82-51-510-0560 (S.K.); +82-51-510-0660 (J.K.); Fax: +82-2-313-7190 (H.L.); $+82-51-510-0568$ (S.K. \& J.K.)

+ These authors contributed equally to this work.

\begin{abstract}
Early diagnosis increases the treatment success rate for active tuberculosis (ATB) and decreases mortality. MicroRNAs (miRNAs) have been studied as blood-based markers of several infectious diseases. We performed miRNA profiling to identify differentially expressed (DE) miRNAs using whole blood samples from 10 healthy controls (HCs), 15 subjects with latent tuberculosis infection (LTBI), and 12 patients with ATB, and investigated the expression of the top six miRNAs at diagnosis and over the treatment period in addition to performing miRNA-target gene network and gene ontology analyses. miRNA profiling identified 84 DE miRNAs in patients with ATB, including 80 upregulated and four downregulated miRNAs. Receiver operating characteristic curves of the top six miRNAs exhibited excellent distinguishing efficiency with an area under curve (AUC) value $>0.85$. Among them, miR-199a-3p and miR-6886-3p can differentiate between ATB and LTBI. Anti-TB treatment restored the levels of miR-199b-3p, miR-199a-3p, miR-16-5p, and miR-374c-5p to HC levels. Furthermore, 108 predicted target genes were related to the regulation of cellular amide metabolism, intrinsic apoptotic signaling, translation, transforming growth factor beta receptor signaling, and cysteine-type endopeptidase activity. The DE miRNAs identified herein are potential biomarkers for diagnosis and therapeutic monitoring in ATB.
\end{abstract}

Keywords: tuberculosis; latent tuberculosis infection; biomarkers; microRNAs

\section{Introduction}

Tuberculosis (TB) is a fatal infectious disease caused by Mycobacterium tuberculosis (MTB) infection [1]. According to the World Health Organization (WHO), there were approximately 10 million new cases and 1.2 million deaths attributed to TB worldwide in 2019 [2]. Approximately $85 \%$ of MTB infections occur in the lungs but infections can spread and affect multiple organs [1]. According to a WHO report in 2021, TB is the most powerful 
single pathogen to have emerged before coronavirus disease 2019 (COVID-19) and about one-quarter of the world's population is infected with MTB. Of all patients infected with MTB, about $5 \%$ of them will have active tuberculosis (ATB), and about $95 \%$ will develop latent TB infection (LTBI). It is likely that they will develop ATB during their lifetime [3].

Early diagnosis and proper therapy can improve the outcomes of patients with ATB. Great advances and efforts have been made in recent years to treat and understand the basal mechanisms of MTB infection [4-6]. However, remission rates remain suboptimal, and TB diagnosis remains challenging, owing to the limitations in the specificity and sensitivity of the current diagnostic tests [7]. There are two typical methods for diagnosing latent MTB infection. The first is the tuberculin skin test (TST), which entails checking the skin reaction to injected antigen, but the reaction is influenced by Bacillus Calmette-Guérin (BCG) vaccination status and non-tuberculous mycobacterial infection, often yielding false results [8]. The second is a commercial interferon gamma (IFN- $\gamma$ ) release assay called the QuantiFERON-TB gold in-tube (QFT-GIT) test method, which is faster and more sensitive than TST but cannot differentiate between patients with ATB and LTBI [9]. Thus, there is a need for a rapid method that accurately distinguishes the disease state with high sensitivity and specificity.

There is a growing interest in microRNAs (miRNAs) as biomarkers of various diseases. miRNAs are short (approximately 22 nucleotides) non-coding RNAs that bind to RNA sequences and perform post-transcriptional gene silencing [10]. When miRNA binds to a complementary sequence in an mRNA molecule, it cuts the mRNA strand, destabilizes the mRNA by shortening the poly(A) tail, or renders the translation of mRNA into protein by ribosomes less efficient, resulting in gene silencing [11,12]. Moreover, miRNAs are involved in the pathogenesis and progression of a wide range of infectious disease processes, including regulating mRNA expression, which modulates innate and adaptive immune responses [13]. Numerous studies have confirmed that miRNAs play important roles in the pathogenesis and progression of $\mathrm{TB}$, and the diagnostic potential of serum miRNA levels has motivated researchers to evaluate their clinical utility in several diseases, including TB and nontuberculous mycobacterial infections [14,15]. Some studies have reported that the host immune response to MTB is modulated by specific miRNAs, and that most are induced by MTB infection [16-22]. Of these, miR-125b and miR-155 were shown to affect an inflammatory response against MTB infection by targeting TNF- $\alpha$ and SHIP1. Moreover, distinct macrophage miRNA expression was elicited according to the virulence of MTB, suggesting an important role for miRNAs in pathogenesis [16,18,23]. Although the function of miRNAs in TB has been established, the potential diagnostic or prognostic value of miRNAs in clinical settings has not been fully investigated.

The effective discovery of diagnostic biomarkers and therapeutic targets has been emerging with the advances in genetic analysis techniques and bioinformatics analysis [24]. Furthermore, miRNAs function in the regulation of multiple genes and modulate activators or suppressors of signaling networks in biological processes [25]. Thus, a comprehensive analysis of miRNAs and the identification of key target genes can provide pivotal opportunities for disease diagnosis and treatment. Herein, we aimed to identify clinically usable differentially expressed (DE) miRNAs using whole blood samples from 10 healthy controls (HCs), 15 individuals with LTBI, and 12 patients with ATB, in order to test the hypothesis that levels of DE miRNAs change over the course of anti-TB drug therapy in patients with ATB. The diagnostic value of DE miRNAs was assessed in terms of sensitivity, specificity, and the area under the curve (AUC) of the receiver operating characteristic (ROC) curve. Finally, we explored the target genes predicted by miRNet to determine their potential roles in TB pathogenesis and disease progression using pathway analysis.

\section{Materials and Methods}

\subsection{Clinical Samples}

Blood samples were collected from Yonsei University Severance Hospital, a tertiary referral hospital in Seoul, Republic of Korea, between January 2016 and September 2019. 
The study was approved by the Institutional Ethics Committee of Yonsei University Severance Hospital (IRB No. 4-2014-1108), and written informed consent was obtained from all participants for the identification of TB biomarkers. Individuals with any acute or chronic disease, previous history of ATB, or symptoms suggestive of TB were excluded. We classified the study participants into an ATB group, an LTBI group, and a healthy control (HC) group [26,27].

1. ATB: patients with ATB were diagnosed based on microbiological and pathological data. A positive MTB culture result from respiratory specimens or the presence of caseous granulomas in lung tissue was used to confirm the diagnosis of ATB. Cases of clinical ATB, which was defined as negative mycobacterial culture findings but favorable clinical and radiological response to anti-TB medication, were also included.

2. LTBI: recent contact with ATB patients testing positive for the QFT-GIT were enrolled. The participants had no history of TB and no suggestive symptoms of TB.

3. HC: the HCs had to be TB-negative based on the QFT-GIT assay and chest x-rays, and no history of TB. They were free of ATB symptoms, and had no recent contact with patients with ATB.

\subsection{AFB Smear and Culture}

For acid-fast bacillus (AFB) detection, auramine-rhodamine fluorescent staining was performed using sputum samples, and the results were confirmed using the Ziehl-Neelsen method $[28,29]$. Mycobacterial culture was conducted using liquid and solid culture media, and the M. tuberculosis complex was confirmed by a duplex PCR using Advansure TB/NTM real-time PCR (LG chemistry, Seoul, Korea). The mycobacterium culture and AFB smear of sputum samples were conducted in a biosafety cabinet class 2 , in compliance with the local and national regulations on containment equipment.

\subsection{QFT-GIT}

The QFT-GIT test, a commercial TB-specific interferon gamma release assay (QIAGEN, Hilden, Germany), was performed according to the manufacturer's instructions. Briefly, $1 \mathrm{~mL}$ of whole blood was collected in three QFT-GIT collection tubes for no stimulation (negative control), MTB-specific peptide stimulation (ESAT-6, CFP-10, and TB7.7), and mitogen stimulation (positive control), and incubated for $16-24 \mathrm{~h}$ at $37^{\circ} \mathrm{C}$. Plasma was harvested by centrifugation at $3000 \times g$ for $15 \mathrm{~min}$ and stored at $-80^{\circ} \mathrm{C}$ until use. The concentration of IFN- $\gamma$ was determined using a QFT-GIT enzyme-linked immunosorbent assay kit according to the manufacturer's instructions. The QFT-GIT test results were interpreted using QFT-GIT software version 2.62 (QIAGEN).

\section{4. miRNA Extraction}

Three milliliters of whole blood were collected into PAXgene Blood RNA tubes (QIAGEN) at diagnosis (T0), at 2 months (T2; during treatment), and at 6-9 months after treatment (T6; completion of treatment), and stored at $-80^{\circ} \mathrm{C}$. miRNAs were extracted using the PAXgene Blood miRNA kit (QIAGEN) according to the manufacturer's instructions. The RNA concentration was measured using a NanoDrop spectrophotometer (Thermo Fisher Scientific, Waltham, MA, USA). The RNA integrity number (RIN) was determined using a BioAnalyzer 2100 (Agilent, Santa Clara, CA, USA), and sequencing was performed using only samples with RIN $\geq 7.0$. The RNA samples were stored at $-80^{\circ} \mathrm{C}$ until use.

\section{5. miRNA Sequence Analysis}

Small RNA libraries were prepared using the NEBNext Multiplex Small RNA Library Prep kit (Illumina, San Diego, CA, USA) according to the manufacturer's instructions. Briefly, an RNA sequencing library was generated on the Illumina NextSeq500 platform via cDNA amplification, end-repair, adenylation of $3^{\prime}$ ends, adapter ligation, and amplification. 


\section{6. miRNA Expression Profiles}

The miRNAs obtained via sequencing were analyzed using MultiExperiment Viewer (MeV 4.9.0; The Perl Foundation, Holland, MI, USA). Hierarchical clustering and volcano plot analyses were performed to identify DE miRNAs. DE miRNAs were identified using cut-off points for the $p$ value $\left(<0.05 ;-\log _{10}(0.05)=1.30\right)$ and mean difference $(>0.5$ or $<-0.5)$.

\section{7. $q R T-P C R$ Validation}

To analyze the expression of DE miRNAs (miR-16-5p, miR-199-3p (detection of both miR-199a-3p and miR-199b-3p), miR-374c-5p, miR-6886-3p, and miR-6856-3p), blood samples after MTB-specific peptide stimulation were treated using $500 \mu \mathrm{L}$ of RNA/DNA stabilization reagent for blood/bone marrow (Roche Diagnostics, Mannheim, Germany). Subsequently, a MagNA Pure LC RNA Isolation Kit-High Performance kit (Roche Diagnostics) was used to extract RNA according to manufacturer's instructions [30].

Next, complementary DNA (cDNA) of miR-16-5p, miR-199a-3p, miR-199b-3p, miR374c-5p, miR-6886-3p, and miR-6856-3p was synthesized using the miRCURY LNA RT Kit (Qiagen) following manufacturer's protocols. The temperature profile for cDNA synthesis reaction was as follows: $42^{\circ} \mathrm{C}$ for $60 \mathrm{~min}$ and $95^{\circ} \mathrm{C}$ for $5 \mathrm{~min}$.

Quantitative reverse transcriptase (qRT)-PCR was performed using the miRCURY LNA miRNA PCR Assay [31]. PCR cycling was performed as follows: $95^{\circ} \mathrm{C}$ for $2 \mathrm{~min}$, followed by 40 cycles of $95^{\circ} \mathrm{C}$ for $10 \mathrm{~s}$ and $56^{\circ} \mathrm{C}$ for $1 \mathrm{~min}$. qRT-PCR were performed on the CFX96 Real-time PCR System Detector (Bio-Rad, Hercules, CA, USA). Samples were run in duplicate for each experiment. To monitor contamination of the reagents, a negative control was included for each primer pair. Data were analyzed using the comparative $\Delta C_{T}$ method $\left(2^{-\Delta C T}\right)$, with RNU44 as an endogenous control [32].

\section{8. miRNA-Target Gene Network Construction and Gene Ontology (GO) Enrichment Analysis}

The web-based platform miRNet (http:/ / www.mirnet.ca/, accessed on 17 December 2021) was used to predict genes targeted by the selected DE miRNAs. The miRNA target gene network was constructed based on the mapping analysis. For GO enrichment analysis, the target genes in the network were analyzed using Cytoscape Version 3.8.1 (Cytoscape Team, Seattle, WA, USA) with ClueGO. ClueGO parameters were set as follows: GO term fusion was selected including the biological process, cellular component, and molecular function; only pathways with $p<0.05$ in Bonferroni step-down analysis were included; kappa score of 0.4 [33].

\subsection{Statistical Analysis}

The data were statistically analyzed using IBM SPSS Statistics for Windows, version 21.0 (IBM, Armonk, NY, USA), GraphPad Prism software Version 6.0 (GraphPad, La Jolla, CA, USA) and MeV 4.9.0 (The Perl Foundation). Comparisons for each group were conducted using the Mann-Whitney $U$ test or one-way analysis of variance test. ROC curve analysis was performed to evaluate the discriminating factors of miRNAs. To assess the diagnostic utility of the DE miRNAs, we investigated the AUC values of the ROC curve, as well as statistical sensitivity and specificity. Statistical significance was set at $p<0.05$.

\section{Results}

\subsection{Characteristics of Study Participants}

A total of 37 study participants including 12 with ATB, 15 with LTBI, and $10 \mathrm{HCs}$ were recruited (Table 1 ). The age (mean \pm standard deviation) of the ATB, LTBI, and HC groups were $41.0 \pm 19.6$ years, $42.1 \pm 15.1$ years, and $34.4 \pm 7.2$ years, respectively. The percentages of male patients in the ATB, LTBI, and HC groups were $66.7 \%(8 / 12), 40.0 \%$ $(6 / 15)$, and $60 \%(6 / 10)$, respectively. BCG scars were present in $9(75.0 \%)$ patients with ATB and 8 (53.3\%) patients with LTBI, and in $9(90.0 \%)$ HCs. There were no significant differences in demographic characteristics among the three groups. 
Table 1. Demographic and clinical characteristics of patients with active tuberculosis, individuals with latent tuberculosis infection, and healthy controls.

\begin{tabular}{ccccc}
\hline Characteristics & ATB $(\boldsymbol{n}=\mathbf{1 2})$ & LTBI $(\boldsymbol{n}=\mathbf{1 5})$ & HC $(\boldsymbol{n}=\mathbf{1 0})$ & $\boldsymbol{p}$ Value \\
\hline Gender (male, $\%)$ & $8(66.7 \%)$ & $6(40.0 \%)$ & $6(60.0 \%)$ & 0.36 \\
Age (mean \pm SD) & $41.0 \pm 19.6$ & $42.1 \pm 15.1$ & $34.4 \pm 7.2$ & 0.44 \\
BCG scar (\%) & $9(75.0 \%)$ & $8(53.3 \%)$ & $9(90.0 \%)$ & 0.15 \\
AFB stain positive & $2(16.7 \%)$ & & & \\
Culture positive & $10(83.3 \%)$ & & & \\
QFT-GIT result & & & & \\
Positive & $12(100.0 \%)$ & $15(100.0 \%)$ & $0(0.0 \%)$ & \\
Intermediate & $0(0.0 \%)$ & $0(0.0 \%)$ & $0(0.0 \%)$ & \\
Negative & $0(0.0 \%)$ & $0(0.0 \%)$ & $10(100.0 \%)$ &
\end{tabular}

Abbreviations: ATB, active tuberculosis; LTBI, latent tuberculosis infection; HC, healthy control; QFT-GIT, QuantiFERON-TB gold in-tube assay.

\subsection{Identification of Potential miRNAs as Diagnostic Biomarker for TB}

To identify miRNAs for potential use as diagnostic biomarkers for TB, miRNA sequencing was performed using total RNA extracted from the blood of study participants, including a complete set of serial samples (T0, T2, and T6) from patients with ATB who underwent a six-month course of treatment. Potential DE miRNAs obtained via miRNA sequencing were selected based on specific criteria (mean difference $>0.5$ or $<-0.5 ; p<0.05$ ), and $84 \mathrm{DE}$ miRNAs, including 80 upregulated and 4 downregulated miRNAs, were identified via volcano plot analysis (Figure 1a). A heat map was generated to classify 84 miRNAs according to their infection status and treatment status (Figure 1b). As the disease progressed (HC to LTBI to ATB), the expression levels of various miRNAs clearly increased or decreased. The top six among them, namely miR-199b-3p, miR-199a-3p, miR-6886-3p, miR-6856-3p, miR-16-5p, and miR-374c-5p, tended to increase as the infection progressed (Figure 2). To validate and confirm whether DE miRNAs are specific to TB, we further performed qRT-PCR verification of the top six DE miRNAs using 51 additional MTB-specific peptide stimulation blood samples (17 HC, 22 LTBI, and 12 ATB samples) (Table S1). miR199a/b-3p, miR-6856-3p, miR-16-5p and miR-374c-5p were observed to be highly expressed in TB samples (Figure S1).

\subsection{Diagnostic Performance of the Top Six DE miRNAs}

To investigate the clinical relevance of the top six DE miRNAs, the AUC, sensitivity, and specificity of miR-199b-3p, miR-199a-3p, miR-6886-3p, miR-6856-3p, miR-16-5p, and miR-374c-5p were analyzed (Table 2). All had AUC values of 0.86 or higher, indicating good diagnostic performance in ATB $(p<0.001)$. In particular, miR-199b-3p showed high sensitivity of $91.67 \%$ and specificity of $90.00 \%$. Table S2 lists the positive and negative results for the top six DE miRNAs along with the coincidence rate with the culture results.

Table 2. The diagnostic utility of differently expressed miRNAs for tuberculosis.

\begin{tabular}{cccccc}
\hline miRNAs & AUC (95\% CI) & Cutoff & Sensitivity (\%) (95\% CI) & Specificity (\%) (95\% CI) & $p$ Value \\
\hline miR-199b-3p & $0.96(0.88-1.03)$ & $>17.50$ & $91.67(61.52-99.79)$ & $90.00 \%(55.50-99.75)$ & $<0.001$ \\
miR-199a-3p & $0.90(0.78-1.03)$ & $>48.00$ & $83.33(51.59-97.91)$ & $90.00 \%(55.50-99.75)$ & $<0.001$ \\
miR-6886-3p & $0.88(0.72-1.03)$ & $>0.50$ & $91.67(61.52-99.79)$ & $80.00 \%(44.39-97.48)$ & $<0.001$ \\
miR-6856-3p & $0.87(0.73-1.02)$ & $>0.50$ & $91.67(61.52-99.79)$ & $60.00 \%(26.24-87.84)$ & $<0.001$ \\
miR-16-5p & $0.87(0.71-1.02)$ & $>2571.00$ & $91.67(61.52-99.79)$ & $80.00 \%(44.39-97.48)$ & $<0.001$ \\
miR-374c-5p & $0.86(0.71-1.01)$ & $>24.50$ & $83.33(51.59-97.91)$ & $80.00 \%(44.39-97.48)$ & $<0.001$ \\
\hline
\end{tabular}

Abbreviations: AUC, area under the receiver operating characteristic curve; $\mathrm{CI}$, confidence interval. 


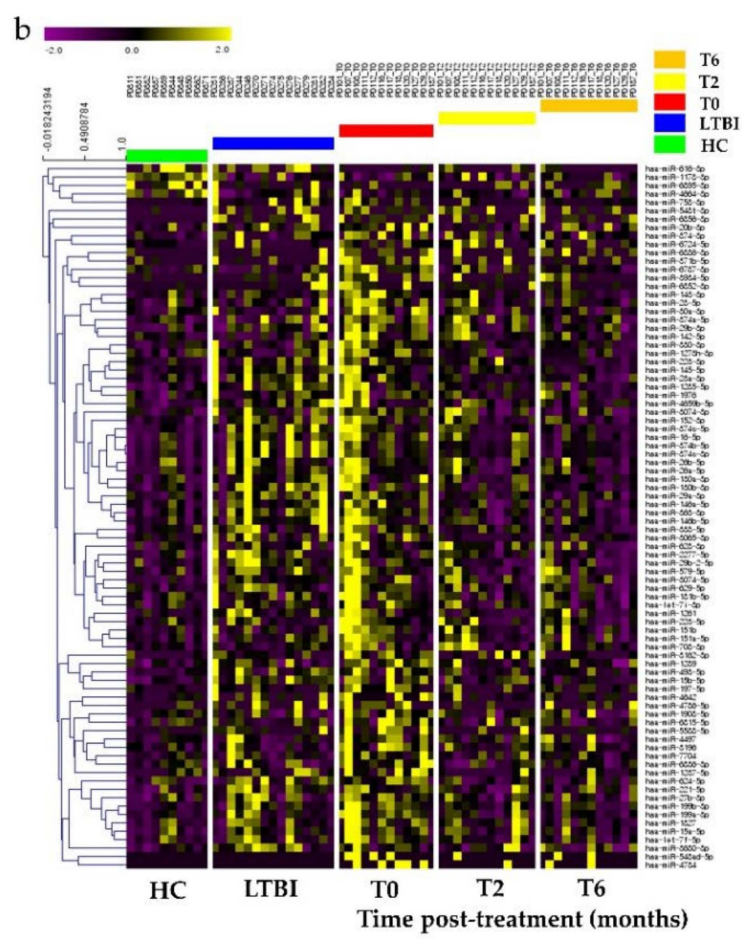

Figure 1. Volcano plot and heat map of differentially expressed miRNAs in whole blood of patients with active tuberculosis (ATB), latent tuberculosis infection (LTBI) and healthy controls (HCs). (a) Volcano plot of miRNAs between patients with ATB and HCs. Cut-off points for the $p$ value $(<0.05$; $\left.-\log _{10}(0.05)=1.30\right)$ or mean difference $(>0.5$ or $<-0.5)$ are indicated by red lines. (b) Heat map of the 84 significantly differentially expressed miRNAs for patients with ATB after two (T2) and six months of treatment (T6), LTBI, and HCs (Pearson correlation; $p<0.05$ by hierarchical clustering analysis). Yellow dots represent upregulated miRNAs, and blue dots represent downregulated miRNAs.

a

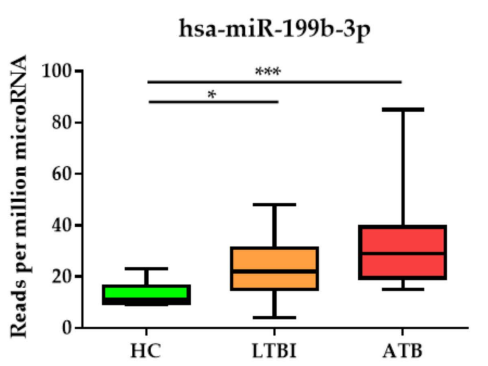

$\mathrm{d}$

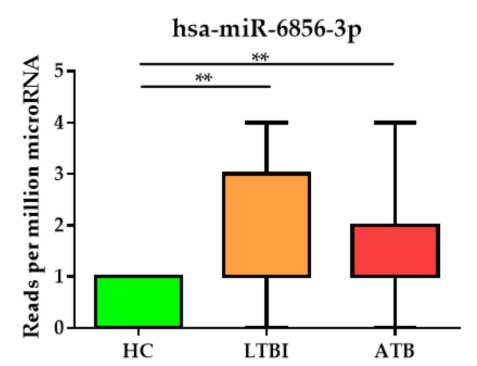

b

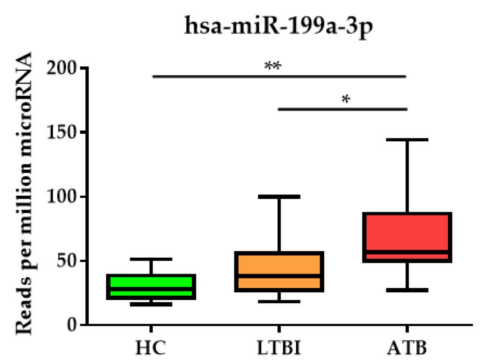

e

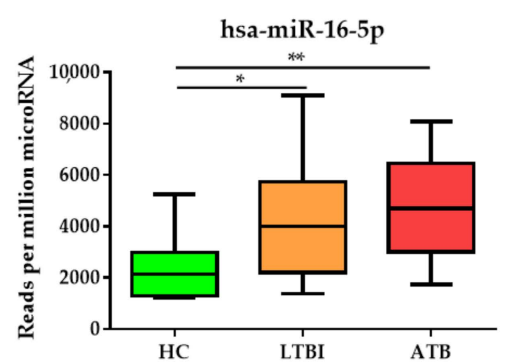

C

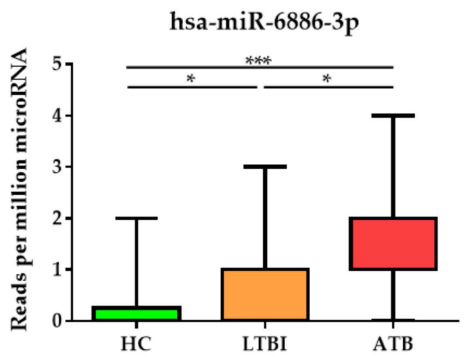

$\mathrm{f}$

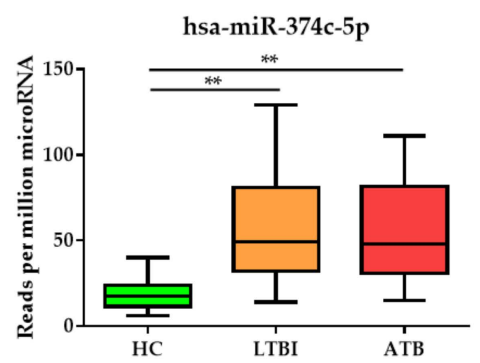

Figure 2. Expression levels of the top six differentially expressed miRNAs, namely (a) miR-199b-3p, (b) miR-199a-3p, (c) miR-6886-3p, (d) miR-6856-3p, (e) miR-16-5p, and (f) miR-374c-5p, in patients with active tuberculosis (ATB), individuals with latent tuberculosis infection (LTBI), and healthy controls (HCs). Data are reported as mean \pm standard deviation. ${ }^{*} p<0.05,{ }^{* *} p<0.01,{ }^{* * *} p<0.001$. 


\subsection{Anti-TB Treatment Response Profiles of Top Six DE miRNAs}

To identify the response to anti-TB therapy in patients with TB during their treatment course, the expression levels of the top six DE miRNAs were assessed after two months of treatment (T2) and again at the end of standard anti-TB therapy, six to nine months after diagnosis (T6). The expression levels of all of the top six DE miRNAs were diminished in patients with ATB after two months and at the end of treatment (Figure 3). In particular, miR-199b-3p, miR-199a-3p, and miR-16-5p levels significantly decreased within 2 months of therapy and continued to trend towards HC levels after completion of therapy.

a

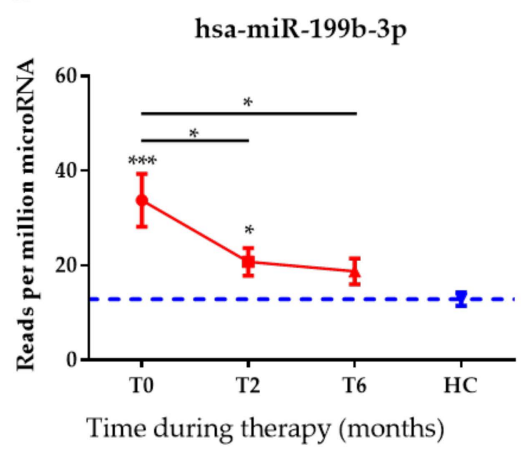

d

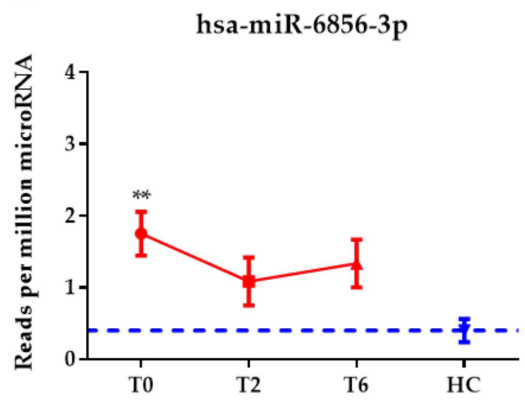

Time during therapy (months) b

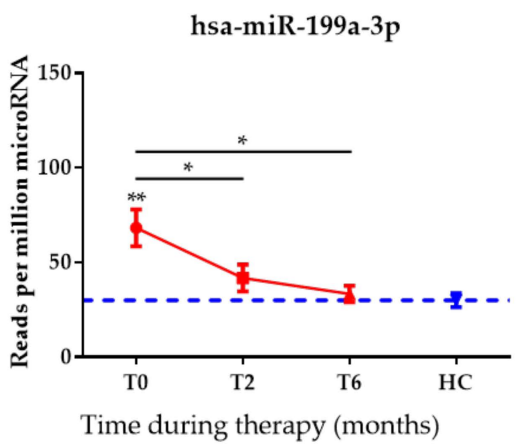

$\mathrm{e}$

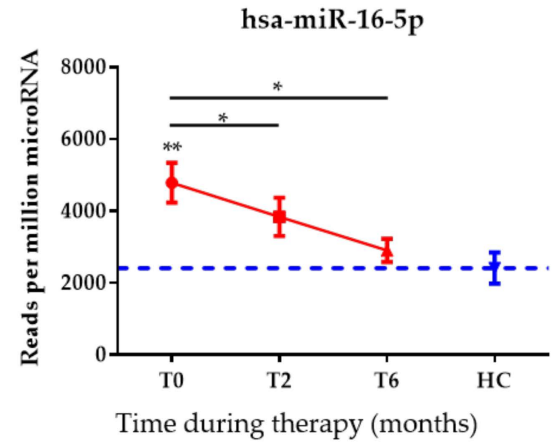

C

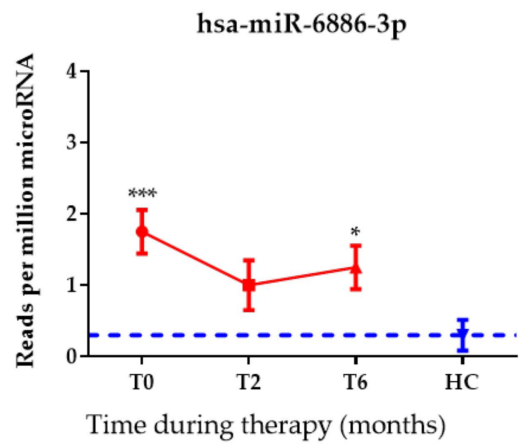

$\mathrm{f}$

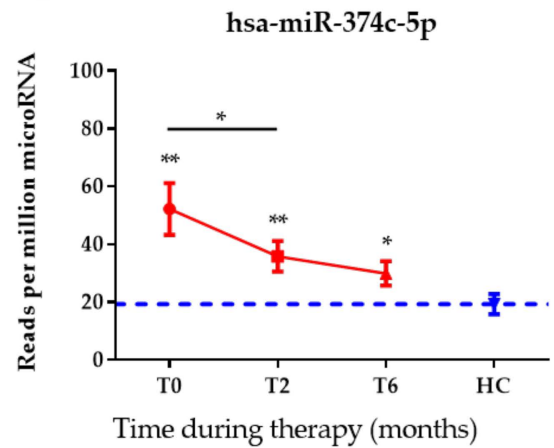

Figure 3. The expression levels of the top six miRNAs in patients with active tuberculosis (ATB) before, during and after treatment. The expression levels of (a) miR-199b-3p, (b) miR-199a-3p, (c) miR-6886-3p, (d) miR-6856-3p, (e) miR-16-5p, and (f) miR-374c-5p were measured in patients with ATB collected at the time of diagnosis (T0), after two months of therapy (T2), and after the completion of therapy (T6; 6-9 months after T0). The blue dotted line is representative of the mean miRNA expression level of the healthy control (HC) patients. The data are shown as mean \pm standard error of the mean. ${ }^{*} p<0.05,{ }^{* *} p<0.001,{ }^{* * *} p<0.0001$.

\subsection{Construction of miRNAs and Target Gene Networks and GO Analysis of Target Genes}

To further investigate the putative biological functions of the top six DE miRNAs, we predicted the target genes of each miRNA using miRNet (http:/ / www.mirnet.ca/, accessed on 17 December 2021). In total, 108 predicted target genes were identified (Table S3). Figure 4a shows the miRNA-target gene network for the top six DE miRNAs.

Subsequently, GO analysis was performed to classify 108 target genes based on biological processes, molecular functions, and cellular components using the ClueGO plug-in of Cytoscape (kappa score $=0.4 ; p<0.05$, Bonferroni step-down analysis) (Figure $4 \mathrm{~b}$ ). The potential target genes were associated with negative regulation of cellular amide metabolic process (GO:0034249), followed by transforming growth factor beta receptor signaling pathway (GO:0007179), negative regulation of translation (GO:0017148), regulation of intrinsic apoptotic signaling pathway (GO:2001242), platelet degranulation (GO:0002576), platelet 
alpha granule (GO:0031091), and double-stranded RNA binding (GO:0003725). The GO analysis of the predicted targets is presented in Table 3 and Table S4.
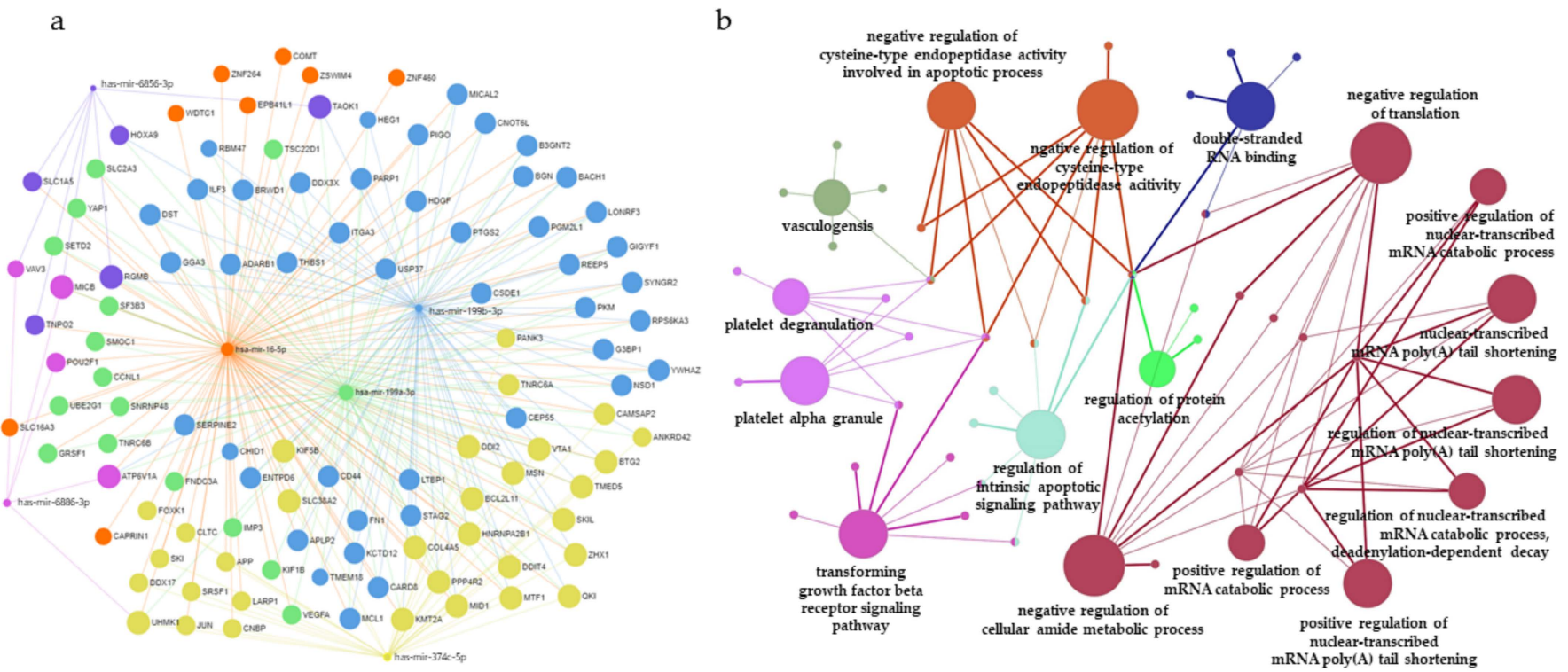

Figure 4. miRNA-mRNA interaction network analysis and gene ontology (GO) enrichment analysis of target genes. (a) The top six miRNAs were uploaded to the miRNet database and. A significant miRNA-target gene network was constructed; miR-199b-3p (blue), miR-16-5p (orange), miR-199a-3p (green), miR-374c-5p (yellow), miR-6856-3p (purple), and miR-6886-3p (pink). (b) GO enrichment analysis of target genes, with the top six differentially expressed miRNAs represented as functionally grouped networks of enriched GO terms generated by ClueGo. The parameters of ClueGO were set as follows: GO term fusion selected; only display GO terms with $p<0.05$ in Bonferroni step-down analysis; kappa score of 0.4 .

Table 3. List of gene ontology terms for predicted target genes of the top six differently expressed miRNAs.

\begin{tabular}{|c|c|c|c|}
\hline GO ID & GO Terms & No. of Genes & $p$ Value \\
\hline GO:0034249 & negative regulation of cellular amide metabolic process & 9 & $<0.001$ \\
\hline GO:0007179 & transforming growth factor beta receptor signaling pathway & 8 & $<0.001$ \\
\hline GO:0017148 & negative regulation of translation & 8 & $<0.001$ \\
\hline GO:2001242 & regulation of intrinsic apoptotic signaling pathway & 7 & $<0.001$ \\
\hline GO:2000117 & negative regulation of cysteine-type endopeptidase activity & 7 & $<0.001$ \\
\hline GO:0043154 & $\begin{array}{l}\text { negative regulation of cysteine-type endopeptidase activity involved in } \\
\text { apoptotic process }\end{array}$ & 6 & $<0.001$ \\
\hline GO:0002576 & platelet degranulation & 6 & $<0.001$ \\
\hline GO:0031091 & platelet alpha granule & 6 & $<0.001$ \\
\hline GO:0001570 & vasculogenesis & 5 & $<0.001$ \\
\hline GO:0003725 & double-stranded RNA binding & 5 & $<0.001$ \\
\hline GO:0061014 & positive regulation of mRNA catabolic process & 4 & $<0.001$ \\
\hline GO:0000289 & nuclear-transcribed mRNA poly(A) tail shortening & 4 & $<0.001$ \\
\hline GO:1901983 & regulation of protein acetylation & 3 & $<0.001$ \\
\hline GO:0060213 & $\begin{array}{l}\text { positive regulation of nuclear-transcribed mRNA poly(A) tail } \\
\text { shortening }\end{array}$ & 3 & $<0.001$ \\
\hline GO:1900151 & $\begin{array}{l}\text { regulation of nuclear-transcribed mRNA catabolic process, } \\
\text { deadenylation-dependent decay }\end{array}$ & 3 & $<0.001$ \\
\hline GO:1900153 & $\begin{array}{l}\text { positive regulation of nuclear-transcribed mRNA catabolic process, } \\
\text { deadenylation-dependent decay }\end{array}$ & 3 & $<0.001$ \\
\hline GO:0060211 & regulation of nuclear-transcribed mRNA poly(A) tail shortening & 3 & $<0.001$ \\
\hline
\end{tabular}




\section{Discussion}

TB is one of the most important infectious diseases, with a high burden of morbidity and mortality [34,35]. Despite the studies aimed at diagnosis and therapy of disease, the development of new screening methods that can detect high-risk conditions and predict treatment responses with easy-to-manage assays is needed for the majority of infected subjects, especially in low-income countries [36]. Currently, many studies are being conducted to improve TB diagnostics, and in particular, diagnostic methods utilizing the immune system are attracting interest [37-39].

miRNAs, also known as epigenetic modulators, participate in many biological processes and pathogenic conditions by regulating post-transcriptional gene expression [40]. Recent studies have found that the expression of specific miRNAs differ according to TB infection status [41]. Studies have been conducted to elucidate the mechanism of miRNAs in several disease processes and whether they hold potential as diagnostic, prognostic, and therapeutic biomarkers $[24,42,43]$. Here, miRNA expression profiling was performed using 37 blood samples consisting of 12 ATB, 15 LTBI, and $10 \mathrm{HC}$ samples to identify potential miRNAs for TB diagnosis and treatment monitoring. Furthermore, a regulatory network was constructed between the identified miRNAs and targets, in addition to a $\mathrm{GO}$ analysis of the predicted target genes. First, our miRNA profiling analysis of $\mathrm{HC}$ and ATB groups identified 84 significant DE miRNAs, including four downregulated miRNAs and 80 upregulated miRNAs. Among these, the top six significant DE miRNAs, namely miR-199b-3p, miR-199a-3p, miR-6886-3p, miR-6856-3p, miR-16-5p, and miR-374c-5p, were significantly upregulated in the blood of patients with ATB compared with HCs. In particular, miR-199a-3p and miR-6886-3p could be used as biomarkers to detect TB infection and to differentiate between ATB and LTBI ( $p<0.05$ and $p<0.05$, respectively). Interestingly, miR-199b-3p, miR-199a-3p, and miR-16 are reportedly related to immune responses and infectious diseases, based on the results of previous studies. It was found that miR-199b-3p expression was increased in septic acute kidney injury models, and that miR-199b-3p binds to nuclear factor erythroid 2-related factor (NRF2), which is a transcription factor directly involved in the transcriptional activation of genes involved in cellular antioxidant responses that plays a central role in the pathogenesis of TB [44,45]. Furthermore, miR-199a-3p can mediate immune tolerance by regulating dendritic cells, leading to increased secretion of interleukin 10 as well as to the inhibition of the phosphatidylinositol 3-kinase/protein kinase $\mathrm{B} /$ nuclear factor kappa B pathway, and miR-16 was significantly upregulated in the serum of patients with TB compared with healthy controls [46,47]. In addition to miR-199b-3p and miR-199a-3p, other miRNAs have also been found to function as major components in cancer biology. Additionally, miR-6886-3p was reported to play a role in hepatocellular carcinoma as a factor that decreases the levels of ubiquitin-specific peptidase 22 [48]. Finally, miR-374c-5p has also been reported to play a role in cancers such as breast cancer $[49,50]$.

Thus, the functions of the most significant DE miRNAs identified herein have been studied previously; however, their clinical relevance has not been fully investigated. Therefore, our study evaluated the potential clinical applications of the top six DE miRNAs in terms of sensitivity, specificity, and AUC of the ROC curve. The results showed a sensitivity of $83.33-91.67 \%$ and a specificity of $60.00-90.00 \%$. All of the top six DE miRNAs showed good ability to efficiently distinguish TB infections from HC with an AUC value greater than 0.85 . The diagnostic values were high or comparable to the previously reported diagnostic values of other miRNAs. Qi et al., reported that the combination of miR-361-5p, miR-889, and miR-576-3p displayed strong potential for diagnostic utility with a high AUC value of 0.863 [51]. Additionally, previous studies have suggested that miR-30c and miR$142-3 p$ serve as diagnostic biomarkers, with AUC values of 0.67 and 0.75 , respectively [52] Jia-Yi Cui et al., reported that AUC values of miR-769-5p, miR-320a, and miR-22-3p range from 0.69 to 0.97 in qRT-PCR validation [53].

Although sputum culture is a standard diagnostic tool for the treatment monitoring of $\mathrm{TB}$, its results are not always indicative of successful treatment [54]. Thus, new biomarkers 
for predicting treatment response are needed for more effective management of patients with TB. In this study, we evaluated the diagnostic utility of the top six miRNAs in patients with $\mathrm{TB}$ who underwent anti-TB drug therapy by comparing the expression levels at time of diagnosis (T0) and after 2 months of intensive anti-TB drug therapy (T2); the results showed that the levels of miR-199b-3p, miR-199a-3p, miR-16-5p, and miR-374c-5p were significantly lower and had reverted to near baseline levels, i.e., to those of HCs. Similar to our findings in the present study, Wagh et al., reported a decrease in the level of miR-16 with treatment [46].

Because miRNAs regulate the expression of target genes to exert their functional features, the accurate prediction of target genes is important. Using miRNet, 108 potential target genes of miR-199b-3p, miR-199a-3p, miR-6886-3p, miR-6856-3p, miR-16-5p, and miR$374 \mathrm{c}-5 \mathrm{p}$ were identified. GO analysis of the identified target genes was performed using ClueGO software. The identified target genes are involved in the negative regulation of cellular amide metabolism, negative regulation of translation, transforming growth factor beta receptor signaling, intrinsic apoptotic signaling pathway, and negative regulation of cysteine-type endopeptidase activity.

This study has several limitations. First, the sample size was small. The expression of the identified DE miRNAs should be further investigated in a larger number of patients from multiple centers. Second, patients with other lung diseases were not included as controls for TB in this study. Further investigation with other lung disease controls is necessary to evaluate the ability of the DE miRNAs to discriminate TB from other lung diseases.

\section{Conclusions}

We suggest that miR-199b-3p, miR-199a-3p, miR-6886-3p, miR-6856-3p, miR-16-5p, and miR-374c-5p can offer promising diagnostic and treatment monitoring markers for TB patients.

Supplementary Materials: The following supporting information can be downloaded at: https: //www.mdpi.com/article/10.3390/diagnostics12020369/s1, Figure S1: Validation of the expression levels of the selected miRNAs among study populations by qRT-PCR; Table S1: Demographic and clinical characteristics of patients with active tuberculosis, individuals with latent tuberculosis, and healthy control for validation of miRNA candidates; Table S2: Positive and negative results for the top six differentially expressed miRNAs; Table S3: The list of predicted target genes; Table S4: The $\mathrm{GO}$ analysis for the predicted targets.

Author Contributions: Conceptualization, H.L., S.K. and J.K. (Jungho Kim); methodology, J.K. (Junseong Kim), H.P., S.-B.P., E.J.L., M.-A.J., E.A. and B.S.; formal analysis, J.K. (Junseong Kim), H.P. and J.L.; writing—original draft preparation, J.K. (Junseong Kim) and H.P.; writing-review and editing, H.J., K.E.L., S.-N.C., Y.A.K., H.L., S.K. and J.K. (Jungho Kim). All authors have read and agreed to the published version of the manuscript.

Funding: This study was supported by the Basic Science Research Program through the National Research Foundation of Korea (NRF) funded by the Ministry of Science, ICT, and Future Planning (NRF-2020R1F1A1052068), and by the Catholic University of Pusan 2021.

Institutional Review Board Statement: The study was approved by the Institutional Ethics Committee of Yonsei University Severance Hospital (IRB No. 4-2014-1108) on 2 May 2018.

Informed Consent Statement: Written informed consent was obtained from all participants for the identification of TB biomarkers.

Data Availability Statement: The data generated or analyzed during this study are included in this published article and its additional files. Some of the datasets are available from the corresponding author upon reasonable request.

Conflicts of Interest: The authors declare no conflict of interest. 


\section{References}

1. Konstantinos, A. Testing for tuberculosis. Aust. Prescr. 2010, 33, 12-18. [CrossRef]

2. WHO. Global Tuberculosis Report 2019; WHO: Geneva, Switzerland, 2019.

3. WHO. Global Tuberculosis Report 2021; WHO: Geneva, Switzerland, 2021.

4. Maitra, A.; Kamil, T.K.; Shaik, M.; Danquah, C.A.; Chrzastek, A.; Bhakta, S. Early diagnosis and effective treatment regimens are the keys to tackle antimicrobial resistance in tuberculosis (TB): A report from Euroscicon's international TB Summit 2016. Virulence 2017, 8, 1005-1024. [CrossRef] [PubMed]

5. Pedersen, J.L.; Bokil, N.J.; Saunders, B.M. Developing new TB biomarkers, are miRNA the answer? Tuberculosis 2019, $118,101860$. [CrossRef] [PubMed]

6. $\quad$ Khader, S.A.; Divangahi, M.; Hanekom, W.; Hill, P.C.; Maeurer, M.; Makar, K.W.; Mayer-Barber, K.D.; Mhlanga, M.M.; Nemes, E.; Schlesinger, L.S.; et al. Targeting innate immunity for tuberculosis vaccination. J. Clin. Investig. 2019, 129, 3482-3491. [CrossRef] [PubMed]

7. Parsons, L.M.; Somoskovi, A.; Gutierrez, C.; Lee, E.; Paramasivan, C.N.; Abimiku, A.; Spector, S.; Roscigno, G.; Nkengasong, J. Laboratory diagnosis of tuberculosis in resource-poor countries: Challenges and opportunities. Clin. Microbiol. Rev. 2011, 24, 314-350. [CrossRef]

8. Bozkanat, E.; Kaya, H.; Sezer, O.; Caliskan, T.; Kilic, E.; Ciftci, F.; Gumus, S.; Kartaloglu, Z. Comparison of tuberculin skin test and quantiferon-TB gold in tube test for diagnosis of latent tuberculosis infection in health care workers: A cross sectional study. $J$. Pak. Med. Assoc. 2016, 66, 270-274.

9. Qiu, X.; Tang, Y.; Zou, R.; Zeng, Y.; Yue, Y.; Li, W.; Qu, Y.; Mu, D. Diagnostic accuracy of interferon-gamma-induced protein 10 for differentiating active tuberculosis from latent tuberculosis: A meta-analysis. Sci. Rep. 2019, 9, 11408. [CrossRef]

10. Bartel, D.P. MicroRNAs: Genomics, biogenesis, mechanism, and function. Cell 2004, 116, 281-297. [CrossRef]

11. Bartel, D.P. MicroRNAs: Target recognition and regulatory functions. Cell 2009, 136, 215-233. [CrossRef]

12. Lewis, B.P.; Burge, C.B.; Bartel, D.P. Conserved seed pairing, often flanked by adenosines, indicates that thousands of human genes are microRNA targets. Cell 2005, 120, 15-20. [CrossRef]

13. Acuna, S.M.; Floeter-Winter, L.M.; Muxel, S.M. MicroRNAs: Biological Regulators in Pathogen-Host Interactions. Cells 2020, 9, 113. [CrossRef] [PubMed]

14. Han, S.A.; Jhun, B.W.; Kim, S.Y.; Moon, S.M.; Yang, B.; Kwon, O.J.; Daley, C.L.; Shin, S.J.; Koh, W.J. miRNA Expression Profiles and Potential as Biomarkers in Nontuberculous Mycobacterial Pulmonary Disease. Sci. Rep. 2020, 10, 3178. [CrossRef] [PubMed]

15. Yuan, Z.; Prasla, Z.; Lee, F.E.; Bedi, B.; Sutliff, R.L.; Sadikot, R.T. MicroRNA-155 Modulates Macrophages' Response to NonTuberculous Mycobacteria through COX-2/PGE2 Signaling. Pathogens 2021, 10, 920. [CrossRef] [PubMed]

16. Rajaram, M.V.; Ni, B.; Morris, J.D.; Brooks, M.N.; Carlson, T.K.; Bakthavachalu, B.; Schoenberg, D.R.; Torrelles, J.B.; Schlesinger, L.S. Mycobacterium tuberculosis lipomannan blocks TNF biosynthesis by regulating macrophage MAPK-activated protein kinase 2 (MK2) and microRNA miR-125b. Proc. Natl. Acad. Sci. USA 2011, 108, 17408-17413. [CrossRef]

17. Afum-Adjei Awuah, A.; Ueberberg, B.; Owusu-Dabo, E.; Frempong, M.; Jacobsen, M. Dynamics of T-cell IFN-gamma and miR-29a expression during active pulmonary tuberculosis. Int. Immunol. 2014, 26, 579-582. [CrossRef]

18. Huang, J.; Jiao, J.; Xu, W.; Zhao, H.; Zhang, C.; Shi, Y.; Xiao, Z. MiR-155 is upregulated in patients with active tuberculosis and inhibits apoptosis of monocytes by targeting FOXO3. Mol. Med. Rep. 2015, 12, 7102-7108. [CrossRef]

19. Song, Q.; Li, H.; Shao, H.; Li, C.; Lu, X. MicroRNA-365 in macrophages regulates Mycobacterium tuberculosis-induced active pulmonary tuberculosis via interleukin-6. Int. J. Clin. Exp. Med. 2015, 8, 15458-15465.

20. Ouimet, M.; Koster, S.; Sakowski, E.; Ramkhelawon, B.; van Solingen, C.; Oldebeken, S.; Karunakaran, D.; Portal-Celhay, C.; Sheedy, F.J.; Ray, T.D.; et al. Mycobacterium tuberculosis induces the miR-33 locus to reprogram autophagy and host lipid metabolism. Nat. Immunol. 2016, 17, 677-686. [CrossRef]

21. Zhang, Z.M.; Zhang, A.R.; Xu, M.; Lou, J.; Qiu, W.Q. TLR-4/miRNA-32-5p/FSTL1 signaling regulates mycobacterial survival and inflammatory responses in Mycobacterium tuberculosis-infected macrophages. Exp. Cell Res. 2017, 352, 313-321. [CrossRef]

22. Zonghai, C.; Tao, L.; Pengjiao, M.; Liang, G.; Rongchuan, Z.; Xinyan, W.; Wenyi, N.; Wei, L.; Yi, W.; Lang, B. Mycobacterium tuberculosis ESAT6 modulates host innate immunity by downregulating miR-222-3p target PTEN. Biochim. Biophys. Acta Mol. Basis Dis. 2022, 1868, 166292. [CrossRef]

23. Abdalla, A.E.; Duan, X.; Deng, W.; Zeng, J.; Xie, J. MicroRNAs play big roles in modulating macrophages response toward mycobacteria infection. Infect. Genet. Evol. 2016, 45, 378-382. [CrossRef] [PubMed]

24. Kim, J. Identification of MicroRNAs as Diagnostic Biomarkers for Breast Cancer Based on the Cancer Genome Atlas. Diagnostics 2021, 11, 107. [CrossRef] [PubMed]

25. Gurtan, A.M.; Sharp, P.A. The role of miRNAs in regulating gene expression networks. J. Mol. Biol. 2013, 425, 3582-3600. [CrossRef] [PubMed]

26. Berry, M.P.; Graham, C.M.; McNab, F.W.; Xu, Z.; Bloch, S.A.; Oni, T.; Wilkinson, K.A.; Banchereau, R.; Skinner, J.; Wilkinson, R.J.; et al. An interferon-inducible neutrophil-driven blood transcriptional signature in human tuberculosis. Nature 2010, 466, 973-977. [CrossRef] 
27. Cho, Y.; Park, Y.; Sim, B.; Kim, J.; Lee, H.; Cho, S.N.; Kang, Y.A.; Lee, S.G. Identification of serum biomarkers for active pulmonary tuberculosis using a targeted metabolomics approach. Sci. Rep. 2020, 10, 3825. [CrossRef]

28. Yates, M.D.; Pozniak, A.; Uttley, A.H.; Clarke, R.; Grange, J.M. Isolation of environmental mycobacteria from clinical specimens in south-east England: 1973-1993. Int. J. Tuberc. Lung Dis. 1997, 1, 75-80.

29. Griffith, D.E.; Aksamit, T.; Brown-Elliott, B.A.; Catanzaro, A.; Daley, C.; Gordin, F.; Holland, S.M.; Horsburgh, R.; Huitt, G.; Iademarco, M.F.; et al. An official ATS/IDSA statement: Diagnosis, treatment, and prevention of nontuberculous mycobacterial diseases. Am. J. Respir. Crit. Care Med. 2007, 175, 367-416. [CrossRef]

30. Kim, S.; Lee, H.; Kim, H.; Kim, Y.; Cho, J.E.; Jin, H.; Kim, D.Y.; Ha, S.J.; Kang, Y.A.; Cho, S.N.; et al. Diagnostic performance of a cytokine and IFN-gamma-induced chemokine mRNA assay after Mycobacterium tuberculosis-specific antigen stimulation in whole blood from infected individuals. J. Mol. Diagn. 2015, 17, 90-99. [CrossRef]

31. Lee, S.; Hong, N.; Kim, Y.; Park, S.; Kim, K.J.; Jeong, J.; Jung, H.I.; Rhee, Y. Circulating miR-122-5p and miR-375 as Potential Biomarkers for Bone Mass Recovery after Parathyroidectomy in Patients with Primary Hyperparathyroidism: A Proof-of-Concept Study. Diagnostics 2021, 11, 1704. [CrossRef]

32. Torres, A.; Torres, K.; Wdowiak, P.; Paszkowski, T.; Maciejewski, R. Selection and validation of endogenous controls for microRNA expression studies in endometrioid endometrial cancer tissues. Gynecol. Oncol. 2013, 130, 588-594. [CrossRef]

33. Bindea, G.; Mlecnik, B.; Hackl, H.; Charoentong, P.; Tosolini, M.; Kirilovsky, A.; Fridman, W.H.; Pages, F.; Trajanoski, Z.; Galon, J ClueGO: A Cytoscape plug-in to decipher functionally grouped gene ontology and pathway annotation networks. Bioinformatics 2009, 25, 1091-1093. [CrossRef] [PubMed]

34. Acharya, B.; Acharya, A.; Gautam, S.; Ghimire, S.P.; Mishra, G.; Parajuli, N.; Sapkota, B. Advances in diagnosis of Tuberculosis: An update into molecular diagnosis of Mycobacterium tuberculosis. Mol. Biol. Rep. 2020, 47, 4065-4075. [CrossRef] [PubMed]

35. Natarajan, A.; Beena, P.M.; Devnikar, A.V.; Mali, S. A systemic review on tuberculosis. Indian J. Tuberc. 2020, 67, $295-311$. [CrossRef] [PubMed]

36. Suarez, I.; Funger, S.M.; Kroger, S.; Rademacher, J.; Fatkenheuer, G.; Rybniker, J. The Diagnosis and Treatment of Tuberculosis Dtsch. Arztebl. Int. 2019, 116, 729-735. [CrossRef] [PubMed]

37. Sinigaglia, A.; Peta, E.; Riccetti, S.; Venkateswaran, S.; Manganelli, R.; Barzon, L. Tuberculosis-Associated MicroRNAs: From Pathogenesis to Disease Biomarkers. Cells 2020, 9, 2160. [CrossRef]

38. Yang, T.; Ge, B. miRNAs in immune responses to Mycobacterium tuberculosis infection. Cancer Lett. 2018, 431, 22-30. [CrossRef]

39. Chen, L.; Heikkinen, L.; Wang, C.; Yang, Y.; Sun, H.; Wong, G. Trends in the development of miRNA bioinformatics tools. Brief. Bioinform. 2019, 20, 1836-1852. [CrossRef]

40. Alipoor, S.D.; Adcock, I.M.; Tabarsi, P.; Folkerts, G.; Mortaz, E. MiRNAs in tuberculosis: Their decisive role in the fate of TB. Eur. J. Pharmacol. 2020, 886, 173529. [CrossRef]

41. Van Rensburg, I.C.; du Toit, L.; Walzl, G.; du Plessis, N.; Loxton, A.G. Decreased neutrophil-associated miRNA and increased B-cell associated miRNA expression during tuberculosis. Gene 2018, 655, 35-41. [CrossRef]

42. Zhou, S.S.; Jin, J.P.; Wang, J.Q.; Zhang, Z.G.; Freedman, J.H.; Zheng, Y.; Cai, L. miRNAS in cardiovascular diseases: Potential biomarkers, therapeutic targets and challenges. Acta Pharmacol. Sin. 2018, 39, 1073-1084. [CrossRef]

43. Shen, S.; Zhang, S.; Liu, P.; Wang, J.; Du, H. Potential role of microRNAs in the treatment and diagnosis of cervical cancer. Cancer Genet. 2020, 248-249, 25-30. [CrossRef]

44. Tian, X.; Liu, Y.; Wang, H.; Zhang, J.; Xie, L.; Huo, Y.; Ma, W.; Li, H.; Chen, X.; Shi, P. The role of miR-199b-3p in regulating Nrf2 pathway by dihydromyricetin to alleviate septic acute kidney injury. Free Radic. Res. 2021, 55, 842-852. [CrossRef] [PubMed]

45. Petrillo, S.; Gallo, M.G.; Santoro, A.; Brugaletta, R.; Nijhawan, P.; Russo, C.; La Rosa, P.; Raponi, M.; Palamara, A.T.; Bertini, E.S.; et al. Personalized profiles of antioxidant signaling pathway in patients with tuberculosis. J. Microbiol. Immunol. Infect. 2021, in press. [CrossRef] [PubMed]

46. Wagh, V.; Urhekar, A.; Modi, D. Levels of microRNA miR-16 and miR-155 are altered in serum of patients with tuberculosis and associate with responses to therapy. Tuberculosis 2017, 102, 24-30. [CrossRef] [PubMed]

47. Xiong, A.; Wang, J.; Mao, X.L.; Jiang, Y.; Fan, Y. MiR-199a-3p modulates the function of dendritic cells involved in transplantation tolerance by targeting CD86. HLA 2019, 94, 493-503. [CrossRef]

48. Xiong, H.; Ni, Z.; He, J.; Jiang, S.; Li, X.; He, J.; Gong, W.; Zheng, L.; Chen, S.; Li, B.; et al. LncRNA HULC triggers autophagy via stabilizing Sirt1 and attenuates the chemosensitivity of HCC cells. Oncogene 2017, 36, 3528-3540. [CrossRef]

49. Hao, S.; Tian, W.; Chen, Y.; Wang, L.; Jiang, Y.; Gao, B.; Luo, D. MicroRNA-374c-5p inhibits the development of breast cancer through TATA-box binding protein associated factor 7-mediated transcriptional regulation of DEP domain containing 1 . J. Cell Biochem. 2019, 120, 15360-15368. [CrossRef]

50. Jee, Y.H.; Wang, J.; Yue, S.; Jennings, M.; Clokie, S.J.; Nilsson, O.; Lui, J.C.; Baron, J. mir-374-5p, mir-379-5p, and mir-503-5p Regulate Proliferation and Hypertrophic Differentiation of Growth Plate Chondrocytes in Male Rats. Endocrinology 2018, 159, 1469-1478. [CrossRef]

51. Qi, Y.; Cui, L.; Ge, Y.; Shi, Z.; Zhao, K.; Guo, X.; Yang, D.; Yu, H.; Cui, L.; Shan, Y.; et al. Altered serum microRNAs as biomarkers for the early diagnosis of pulmonary tuberculosis infection. BMC Infect. Dis. 2012, 12, 384. [CrossRef]

52. Gao, S.H.; Chen, C.G.; Zhuang, C.B.; Zeng, Y.L.; Zeng, Z.Z.; Wen, P.H.; Yu, Y.M.; Ming, L.; Zhao, J.W. Integrating serum microRNAs and electronic health records improved the diagnosis of tuberculosis. J. Clin. Lab. Anal. 2021, 35, e23871. [CrossRef] 
53. Cui, J.Y.; Liang, H.W.; Pan, X.L.; Li, D.; Jiao, N.; Liu, Y.H.; Fu, J.; He, X.Y.; Sun, G.X.; Zhang, C.L.; et al. Characterization of a novel panel of plasma microRNAs that discriminates between Mycobacterium tuberculosis infection and healthy individuals. PLoS ONE 2017, 12, e0184113. [CrossRef] [PubMed]

54. Kim, S.Y.; Kim, J.; Kim, D.R.; Kang, Y.A.; Bong, S.; Lee, J.; Kim, S.; Lee, N.S.; Sim, B.; Cho, S.N.; et al. Urine IP-10 as a biomarker of therapeutic response in patients with active pulmonary tuberculosis. BMC Infect. Dis. 2018, 18, 240. [CrossRef] [PubMed] 